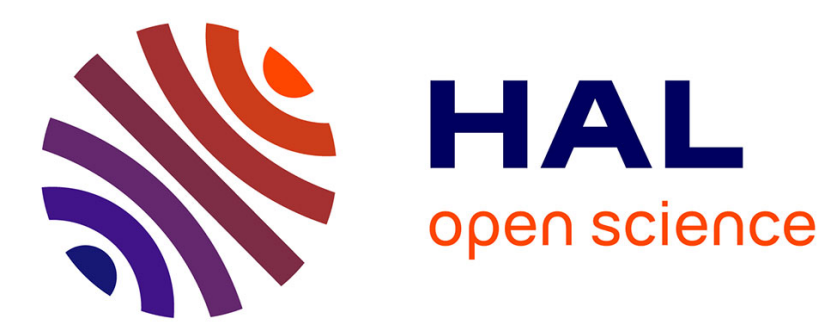

\title{
New nonlinear multiscale models for wrinkled membranes
}

\author{
Noureddine Damil, Michel Potier-Ferry, Heng Hu
}

\section{To cite this version:}

Noureddine Damil, Michel Potier-Ferry, Heng Hu. New nonlinear multiscale models for wrinkled membranes. 2013. hal-00823921

\author{
HAL Id: hal-00823921 \\ https://hal.science/hal-00823921 \\ Preprint submitted on 19 May 2013
}

HAL is a multi-disciplinary open access archive for the deposit and dissemination of scientific research documents, whether they are published or not. The documents may come from teaching and research institutions in France or abroad, or from public or private research centers.
L'archive ouverte pluridisciplinaire HAL, est destinée au dépôt et à la diffusion de documents scientifiques de niveau recherche, publiés ou non, émanant des établissements d'enseignement et de recherche français ou étrangers, des laboratoires publics ou privés. 


\title{
New nonlinear multiscale models for wrinkled membranes
}

\author{
Noureddine Damil ${ }^{\mathrm{a}}$, Michel Potier-Ferry ${ }^{\mathrm{b}, \mathrm{c}}$, Heng $\mathrm{Hu}^{\mathrm{d}}$
}

\begin{abstract}
a) Laboratoire d'Ingénierie et Matériaux LIMAT, Faculté des Sciences Ben M'Sik, Université Hassan II Mohammedia - Casablanca, Sidi Othman, Casablanca, Maroc. noureddine.damil@gmail.com
\end{abstract}

b) LEM3, Laboratoire d'Etudes des Microstructures et de Mécanique des Matériaux, UMR CNRS 7239, Université de Lorraine, Ile du Saulcy, 57045 Metz Cedex 01, France. michel.potier-ferry@univ-lorraine.fr

c) Laboratory of Excellence on Design of Alloy Metals for low-mAss Structures (DAMAS), Université de Lorraine, France

d) School of Civil Engineering, Wuhan University, 8 South Road of East Lake, 430072 Wuhan, P.R. China.

huheng@whu.edu.cn

\begin{abstract}
:
A new macroscopic approach to modelise membrane wrinkling is presented. Most of the studies of the literature about membrane behaviour are macroscopic and phenomenological, the influence of wrinkles being accounted by non-linear constitutive laws without compressive stiffness. The present method is multi-scale and it permits to predict the wavelength and the spatial distribution of wrinkling amplitude. It belongs to the family of Landau-Ginzburg bifurcation equations and especially it relies on the technique of Fourier series with slowly varying coefficients. The result is a new family of macroscopic membrane models that are deduced from Föppl-Von Karman plate equations. Numerical solutions are presented giving the size of the wrinkles as a function of the applied compressive and tensile stresses.
\end{abstract}

Keywords: Wrinkling, membrane, slowly variable Fourier coefficients, multi-scale

\section{Introduction}

Two main classes of numerical approaches are currently used to modelise membrane mechanical behaviour and wrinkling. The first class of methods is based on elastic shell models; see for instance $[1,2,3,4]$. Nowadays many commercial finite element codes permit us to carry out such non-linear shell computations. The advantage of shell analyses is their capacity to describe the details of the membrane response: instability threshold, size, wavelength and orientation of the wrinkles...As a counterpart, the numerical model is heavy and especially very difficult to be controlled in cases with many wrinkles, what leads generally to many equilibrium solutions. These full models will be referred as "microscopic models" because their finite element discretisation provides a detailed response at the scale of the wrinkles.

With the second group of numerical methods, one does not intend to fully describe the wrinkles, but only the decrease of stress they generate. The bending stiffness is neglected and the wrinkling is accounted indirectly by a non-linear constitutive law of unilateral type, where the compressive stresses are eliminated [5,6]. Two variants have to be mentioned: first the 
method of Roddeman [7] that splits the deformation gradient into consistent membrane part and wrinkling part $[8,9,10,11]$; second models with an internal length, like Cosserat theory $[12,13]$ that avoid loss of ellipticity in case of compressive stresses. These models can be considered as macroscopic ones and indeed they require much less refined meshes as the previous ones, because the size of the macroscopic finite elements is not related to size of the wrinkles.

In this paper, macroscopic models of membranes including wrinkling are deduced from the fine plate model without any phenomenological assumptions. The bending stiffness effects are included, not only to define the wrinkling wavelength, but also to predict the macroscopic evolution of the wrinkling pattern. The idea is to build a theory coupling pure membrane model with an envelope equation as in Landau-Ginzburg approach $[14,15]$. Nevertheless we shall not apply the classical Landau-Ginzburg asymptotic technique that is valid only near the bifurcation, but a variant where the nearly periodic fields are represented by Fourier series with slowly varying coefficients $[16,17,18,19]$. In other words we use a multi-scale method whose result is a generalised continuum including an internal length and where the macroscopic stresses are Fourier coefficients of the microscopic stress. The resulting models are macroscopic and require only rough meshes because their unknowns are in-plane displacements and slowly varying envelopes of the wrinkles.

For simplicity, we limit ourselves to plane membrane, the fine model being the traditional Föppl-Von Karman plate equations. Two examples assess model's ability to represent the behaviour of membranes in the presence of wrinkling.

\section{Nonlinear macroscopic models of wrinkling}

\subsection{The full model}

The well known Föppl -Von Karman equations for elastic isotropic plates will be considered as the reference model in this paper:

$$
\left\{\begin{array}{c}
\mathrm{D} \Delta^{2} \mathrm{~W}-\operatorname{div}(\mathbf{N} \nabla \mathrm{w})=0 \\
\mathbf{N}=\mathbf{L}^{\mathbf{m}} \cdot \boldsymbol{\gamma} \\
2 \gamma=\nabla \mathbf{u}+{ }^{\mathrm{t}} \nabla \mathbf{u}+\nabla \mathbf{u} \otimes \nabla \mathbf{u} \\
\operatorname{div} \mathbf{N}=0
\end{array}\right.
$$

where $\mathbf{u}=(\mathrm{u}, \mathrm{v}) \in \mathrm{IR}^{2}$ is the in-plane displacement, $\mathrm{w}$ is the deflection, $\mathbf{N}$ and $\gamma$ are the membrane stress and strain. With the vectorial notations $\left(\begin{array}{l}\mathbf{N} \rightarrow \\ \rightarrow^{t}\left(N_{X}\right.\end{array} N_{Y} \quad N_{X Y}\right)$, $\left.\gamma \rightarrow^{t}\left(\begin{array}{lll}\gamma_{\mathrm{X}} & \gamma_{\mathrm{Y}} & 2 \gamma_{\mathrm{XY}}\end{array}\right)\right)$, the membrane elasticity tensor is represented by the matrix $\frac{\text { Eh }}{1-v^{2}}\left[\begin{array}{ccc}1 & v & 0 \\ v & 1 & 0 \\ 0 & 0 & \frac{1-v}{2}\end{array}\right]$. The corresponding energy $\mathcal{E}$ can be split into a membrane part $\mathcal{E}_{\text {mem }}$ and a bending part $\mathcal{E}_{b e n}$, as follows: 


$$
\left\{\begin{array}{c}
\mathcal{E}(\mathbf{u}, w)=\mathcal{E}_{b e n}(w)+\mathcal{E}_{\text {mem }}(\mathbf{u}, w) \\
2 \mathcal{E}_{b e n}(w)=D \iint\left(\Delta^{2} w-2(1-v)\left(\frac{\partial^{2} w}{\partial X^{2}} \frac{\partial^{2} w}{\partial Y^{2}}-\left(\frac{\partial^{2} w}{\partial X \partial Y}\right)^{2}\right)\right) d \omega \\
2 \mathcal{E}_{m e m}(\mathbf{u}, w)=\iint{ }^{t} \gamma \cdot \mathbf{L}^{m} \cdot \gamma d w=\frac{E h}{1-v^{2}} \iint\left(\gamma_{X}^{2}+\gamma_{Y}^{2}+2(1-v) \gamma_{X Y}^{2}+2 v \gamma_{X} \gamma_{Y}\right) d \omega
\end{array} .\right.
$$

\subsection{A multiscale approach using Fourier coefficient}

We adapt in this 2D framework the method of Fourier series with slowly variables coefficients [17]. For simplicity, we suppose that the instability wavenumber $\mathrm{Q}$ is known and we only consider wrinkles in the OY-direction. Within this method, the unknown field $\mathbf{U}(X, Y)=(u(X, Y) \quad v(X, Y) \quad n(X, Y) \quad \gamma(X, Y))$, whose components are axial displacement, transverse displacement, membrane stress and strains, is written in the following form:

$$
\mathbf{U}(X, Y)=\sum_{m=-\infty}^{+\infty} \mathbf{U}_{m}(X, Y) \exp (i m Q X)
$$

where the new macroscopic unknown fields $\mathbf{U}_{m}(X, Y)$ vary slowly on a single period $\left[X, X+\frac{2 \pi}{Q}\right]$ of the oscillation pattern. Of course we do not need an infinite number of Fourier coefficients and we limit ourselves to three harmonics: the mean field $\mathbf{U}_{0}(X, Y)$ and the envelope of the oscillations $\mathbf{U}_{1}(\mathrm{X}, \mathrm{Y}) \mathrm{e}^{\mathrm{iQX}}, \overline{\mathbf{U}}_{1}(\mathrm{X}, \mathrm{Y}) \mathrm{e}^{\mathrm{i} \mathrm{iQX}}$. According to (Damil and PotierFerry 2010), the second harmonic should be taken into account to recover the results of the Landau-Ginzburg bifurcation approach. Nevertheless the rapid one-dimensional oscillations $\mathrm{e}^{\mathrm{iQX}}$ are inextensional so that they do not contribute to the membrane energy. The macroscopic model with the second harmonic has been established, but within the approximation of ([17], §4.1), we have shown that $\mathbf{N}_{2}=0, \mathrm{w}_{2}=0$ so that the second harmonic does not influence the macroscopic model. The details of this calculation are omitted.

In principle, the mean field $\mathbf{U}_{0}(X, Y)$ is real and the envelope $\mathbf{U}_{1}(X, Y)$ is complex-valued, but spatial evolutions of the patterns can be reasonably accounted with only two real coefficients: for practical finite element calculations, this simplification of two real unknowns will be done, even if a complex envelope can improve the treatment of boundary conditions [19].

The derivation rules are straightforward [16] and in a first time, the derivatives of the envelopes are not neglected. For instance the first Fourier coefficient of the gradient of the deflection is given by:

$$
\left\{(\nabla \mathrm{w})_{1}\right\}=\left\{\begin{array}{c}
\frac{\partial \mathrm{w}_{1}}{\partial \mathrm{X}}+\mathrm{iQw}_{1} \\
\frac{\partial \mathrm{w}_{1}}{\partial \mathrm{Y}}
\end{array}\right\}
$$




\subsection{Macroscopic membrane energy}

We now derive the macroscopic model and we begin with the membrane effects. We apply the principles established in [16][17]. The derivatives are computed exactly as in (4), but some simplifications will be added to obtain the simplest macroscopic model having the same internal length as the asymptotic Landau-Ginzburg approach. Next one could deduce the macroscopic model by identifying Fourier coefficients in the differential equations (1), but a most convenient approach consists in retaining only the harmonic of level zero to approximate an energy density:

$$
\iint_{\text {period }} h d \omega \approx \iint_{\text {period }} h_{0} d \omega
$$

where $h_{0}$ represents the harmonic zero of the density $h$. The rule (5) is a consequence of the assumption of slowly varying envelopes. For instance the energy due a higher harmonic vanishes if the envelope is assumed to be constant on a period:

$$
\iint_{\text {period }} h_{n}(X, Y) \exp (\operatorname{in} Q x) d \omega \approx h_{n}(X, Y) \iint_{\text {period }} \exp (\operatorname{in} Q x) d \omega=0
$$

First, we compute the mean value or the harmonic zero of the Lagrange strain, by using (4) and without any approximation:

$$
\left\{\gamma_{0}\right\}=\left\{\begin{array}{c}
\gamma_{\mathrm{X}_{0}} \\
\gamma_{\mathrm{Y}_{0}} \\
2 \gamma_{\mathrm{XY}_{0}}
\end{array}\right\}=\left\{\begin{array}{c}
\frac{\partial \mathrm{u}_{0}}{\partial \mathrm{X}}+\frac{1}{2}\left(\frac{\partial \mathrm{w}_{0}}{\partial \mathrm{X}}\right)^{2}+\left|\frac{\partial \mathrm{w}_{1}}{\partial \mathrm{X}}+\mathrm{iQ \textrm {w } _ { 1 }}\right|^{2} \\
\frac{\partial \mathrm{v}_{0}}{\partial \mathrm{Y}}+\frac{1}{2}\left(\frac{\partial \mathrm{w}_{0}}{\partial \mathrm{Y}}\right)^{2}+\left|\frac{\partial \mathrm{w}_{1}}{\partial \mathrm{Y}}\right|^{2} \\
\frac{\partial \mathrm{u}_{0}}{\partial \mathrm{Y}}+\frac{\partial \mathrm{v}_{0}}{\partial \mathrm{X}}+\frac{\partial \mathrm{w}_{0}}{\partial \mathrm{X}} \frac{\partial \mathrm{w}_{0}}{\partial \mathrm{Y}}+\left(\frac{\partial \mathrm{w}_{1}}{\partial \mathrm{X}}+\mathrm{iQ} \mathrm{w}_{1}\right) \frac{\partial \overline{\mathrm{w}}_{1}}{\partial \mathrm{Y}}+\left(\frac{\partial \overline{\mathrm{w}}_{1}}{\partial \mathrm{X}}-\mathrm{iQ}_{1}\right) \frac{\partial \mathrm{w}_{1}}{\partial \mathrm{Y}}
\end{array}\right\} .
$$

Next two additional simplifications will be introduced in the envelope model, in the same spirit as in [17]. First the displacement field is reduced to a membrane mean displacement and to a bending wrinkling, i.e. $\mathbf{u}_{1}=0, \mathrm{w}_{0}=0$, which means that we only consider the influence of wrinkling on a flat membrane state. Second the deflection envelope is assumed to be real, which disregards the phase modulation of the wrinkling pattern: $\mathrm{w}_{1}(\mathrm{X}, \mathrm{Y})$ is real. Hence the envelope of the displacement is reduced to three components $\mathbf{u}_{0}=\left(\mathrm{u}_{0}, \mathrm{v}_{0}\right)$ and $\mathrm{w}_{1}$ that will be rewritten for simplicity as $(\mathrm{u}, \mathrm{v}) \stackrel{\text { def }}{=}\left(\mathrm{u}_{0}, \mathrm{v}_{0}\right), \mathrm{def}=\mathrm{w}_{1}$. Hence the simplified version of the mean strain field is: 


$$
\{\gamma\} \stackrel{\text { def }}{=}\left\{\gamma_{0}\right\}=\left\{\begin{array}{c}
\frac{\partial \mathrm{u}}{\partial \mathrm{X}}+\left(\frac{\partial \mathrm{w}}{\partial \mathrm{X}}\right)^{2}+\mathrm{Q}^{2} \mathrm{w}^{2} \\
\frac{\partial \mathrm{v}}{\partial \mathrm{Y}}+\left(\frac{\partial \mathrm{w}}{\partial \mathrm{Y}}\right)^{2} \\
\frac{\partial \mathrm{u}}{\partial \mathrm{Y}}+\frac{\partial \mathrm{v}}{\partial \mathrm{X}}+2 \frac{\partial \mathrm{w}}{\partial \mathrm{X}} \frac{\partial \mathrm{w}}{\partial \mathrm{Y}}
\end{array}\right\} .
$$

The membrane strain formula (8) is quite similar to the strain of the initial Von Karman model. It can be split, first in a linear part $\varepsilon(\mathbf{u})$ that is the symmetric part of the displacement gradient and corresponds to the pure membrane strain, second to a nonlinear part $\gamma^{\mathrm{wr}}(\mathrm{w})$ that is more or less equivalent to wrinkling deformation of [7] and is given by

$$
\left\{\gamma^{w r}(w)\right\}=\left\{\begin{array}{c}
\left(\frac{\partial w}{\partial X}\right)^{2}+Q^{2} w^{2} \\
\left(\frac{\partial w}{\partial Y}\right)^{2} \\
2 \frac{\partial w}{\partial X} \frac{\partial w}{\partial Y}
\end{array}\right\} .
$$

The main difference with the classical Von Karman strain is the extension $\mathrm{Q}^{2} \mathrm{w}^{2}$ in the direction of the wrinkles. So, if the linear strain is compressive, the wrinkling leads to a decrease of the membrane strain.

Last we apply the multiple scale rule (5) to the membrane energy. This leads for instance to

$$
\iint_{\text {period }} \gamma_{X}^{2} d \omega \approx \iint_{\text {period }}\left(\gamma_{X 0}^{2}+2 \gamma_{X 1}^{2}\right) d \omega \approx \iint_{\text {period }} \gamma_{X 0}^{2} d \omega
$$

where the first approximation is deduced from (5) and from Parseval identity, truncated at harmonic one and the second one follows from the assumption $\mathbf{u}_{1}=0, \mathrm{w}_{0}=0$. According to these rules, the membrane energy becomes:

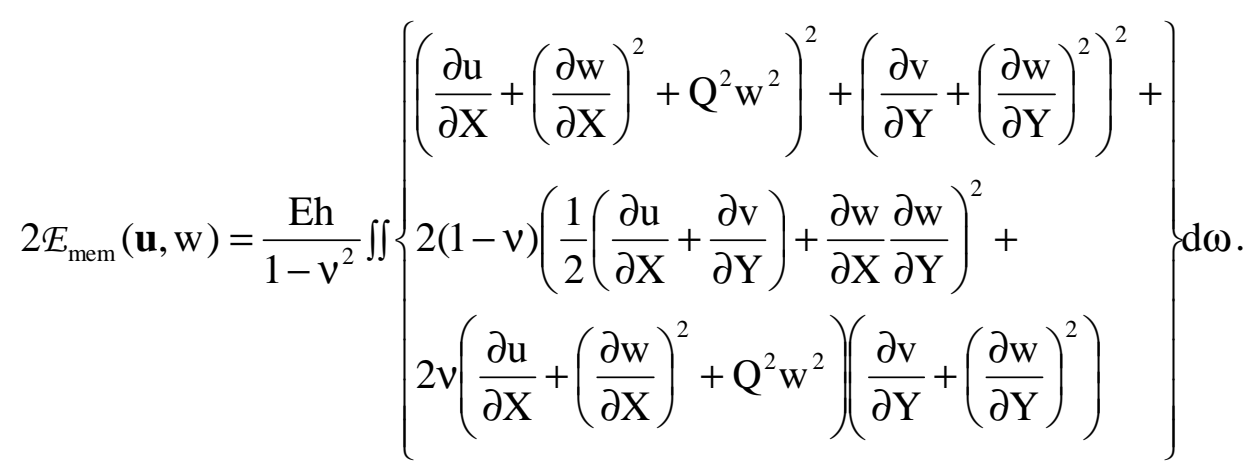




\subsection{Macroscopic bending energy}

The bending energy is reduced to a macroscopic version in the same framework: $\mathbf{u}_{1}=\left(\mathrm{u}_{1}, \mathrm{v}_{1}\right)=(0,0), \mathrm{w}_{0}=0, \mathrm{w}_{1}$ real. The identity (5) is applied to the two terms of the bending energy

$$
h=(\Delta w)^{2}-2(1-v)\left[\frac{\partial^{2} w}{\partial X^{2}} \frac{\partial^{2} w}{\partial Y^{2}}-\left(\frac{\partial^{2} w}{\partial X \partial Y}\right)^{2}\right]=h^{A}-2(1-v) h^{B} .
$$

Hence

$$
\begin{aligned}
\mathrm{h}_{0}^{\mathrm{A}} & =2(\Delta \mathrm{w})_{1}(\Delta \mathrm{w})_{-1}=2\left|(\Delta \mathrm{w})_{1}\right|^{2} \\
& =2\left|\Delta \mathrm{w}_{1}-\mathrm{Q}^{2} \mathrm{w}_{1}+2 \mathrm{iQ} \frac{\partial \mathrm{w}_{1}}{\partial \mathrm{X}}\right|^{2}
\end{aligned}
$$

Since $\mathrm{w}=\mathrm{w}_{1}$ is real, one gets:

$$
\mathrm{h}_{0}^{\mathrm{A}}=2\left(\Delta \mathrm{w}-\mathrm{Q}^{2} \mathrm{w}\right)^{2}+8 \mathrm{Q}^{2}\left(\frac{\partial \mathrm{w}}{\partial \mathrm{X}}\right)^{2}
$$

In the same way, the second term $\mathrm{h}_{0}^{\mathrm{B}}$ is given by:

$$
\mathrm{h}_{0}^{\mathrm{B}}=2\left(\frac{\partial^{2} \mathrm{w}}{\partial \mathrm{X}^{2}}-\mathrm{Q}^{2} \mathrm{w}\right) \frac{\partial^{2} \mathrm{w}}{\partial \mathrm{Y}^{2}}-2\left(\frac{\partial^{2} \mathrm{w}}{\partial \mathrm{X} \partial \mathrm{Y}}\right)^{2}-2 \mathrm{Q}^{2}\left(\frac{\partial \mathrm{w}}{\partial \mathrm{Y}}\right)^{2}
$$

As in ([17], §4.3), the derivatives of order three or four in the differential equations are neglected because the derivatives of order two are sufficient to define a macroscopic length scale and to recover the Landau-Ginzburg asymptotic approach. This leads to

$$
\mathcal{E}_{\text {ben }}(\mathrm{w})=\iint\left\{\mathrm{Q}^{4} \mathrm{w}^{2}-2 \mathrm{Q}^{2} \mathrm{w} \Delta \mathrm{w}+4 \mathrm{Q}^{2}\left(\frac{\partial \mathrm{w}}{\partial \mathrm{X}}\right)^{2}+2\left(1-\mathrm{v}^{2}\right) \mathrm{Q}^{2}\left[\mathrm{w} \frac{\partial^{2} \mathrm{w}}{\partial \mathrm{Y}^{2}}+\left(\frac{\partial \mathrm{w}}{\partial \mathrm{Y}}\right)^{2}\right]\right\} \mathrm{d} \omega
$$

\subsection{The full membrane wrinkling model}

The macroscopic membrane model is deduced from the total energy that is the sum of the membrane energy (11), of the bending energy (14) and of the energy of the applied loads. Let us calculate the corresponding partial differential equations in the case where all the external loads are apply on the boundary. In this case, the sum of bending and membrane energies is stationary

$$
\delta \mathcal{E}_{\text {ben }}+\delta \mathcal{E}_{\text {mem }}=0
$$

for any virtual displacement that is zero at the boundary. This gives: 


$$
\begin{aligned}
& \delta \mathcal{E}_{\text {ben }}+\iint_{\omega} \mathbf{N}: \delta \boldsymbol{\gamma}^{\mathrm{wr}} \mathrm{d} \omega=0 \\
& \iint_{\omega} \mathbf{N}: \delta \boldsymbol{\varepsilon} \mathrm{d} \omega=0 .
\end{aligned}
$$

After straightforward calculations, the differential equations of the macroscopic problem are the followings:

$$
\begin{aligned}
& \operatorname{div} \mathbf{N}=0 \\
& \mathbf{N}=\mathbf{L}^{\mathrm{m}}:\left[\boldsymbol{\varepsilon}(\mathrm{u})+\boldsymbol{\gamma}^{\mathrm{wr}}(\mathrm{w})\right] \\
& -6 \mathrm{DQ}^{2} \frac{\partial^{2} \mathrm{w}}{\partial \mathrm{X}^{2}}-2 \mathrm{DQ}^{2} \frac{\partial^{2} \mathrm{w}}{\partial \mathrm{Y}^{2}}+\left(\mathrm{DQ}^{4}+\mathrm{N}_{\mathrm{X}} \mathrm{Q}^{2}\right) \mathrm{w}-\operatorname{div}(\mathbf{N} \cdot \boldsymbol{\nabla} \mathrm{w})=0,
\end{aligned}
$$

where the expression of the wrinkling membrane strain $\gamma^{\mathrm{wr}}(\mathrm{w})$ is given in (9).

\subsection{Comments}

1. The nonlinear model (17) (18) (19) couples nonlinear membrane equations with a bifurcation equation (19) satisfied by the envelope of wrinkling patterns. It extends the analysis of [17] that coupled a beam membrane with a one-dimensional LandauGinzburg equation. Hence the bifurcation equation (19) is a sort of bi-dimensional Landau-Ginzburg equation.

2. If the membrane stress is prescribed and uniform $\mathbf{N}=\mathrm{N}_{\mathrm{X}} \mathbf{e}_{\mathrm{X}} \otimes \mathbf{e}_{\mathrm{X}}+\mathrm{N}_{\mathrm{Y}} \mathbf{e}_{\mathrm{Y}} \otimes \mathbf{e}_{\mathrm{Y}}$,

(19) becomes identical to a linear eigenvalue problem as in a linear buckling analysis. In the general case, the membrane stress is unknown and (17) (18) (19) is a nonlinear coupled membrane-wrinkling model that can be solved by standard numerical techniques. In this paper, an example of numerical solution is presented in Part 3.2.

3. The nonlinear model (17)(18)(19) is consistent with two ideas used in other macroscopic membrane models. First we have obtained a splitting between a membrane strain and a wrinkling strain, as in the well known theory by Roddeman et al [7]. Next, since we follow an approach consistent with Landau-Ginzburg theory, the final bifurcation equation (19) includes an internal length. As underlined in [16], the mechanical model is a generalized continuum and the stress is not reduced to the mean value of microscopic stress: the first terms of (19) contains the effect of the first Fourier coefficient of the bending moment. This can be qualitatively compared with the ideas of Banerjee et al $[12,13]$ who introduce an internal length via Cosserat theory.

4. The differential equations (17) (18) (19) seem quite different from the classical pure membrane theory that postulates a nonlinear relation between membrane stress and strain; see for instance [5]. Nevertheless the pure membrane theory can be consistent with a degenerate version of (17) (18) (19). The latter can be obtained by dropping all derivatives in (19) which leads to $\left(\mathrm{N}_{\mathrm{X}}+\mathrm{DQ}^{2}\right) \mathrm{w}=0$. If one transforms the latter in a perturbed bifurcation equation as: 


$$
\left(N_{X}+D Q^{2}\right) w=\delta
$$

one gets the deflection as a function of one component of the membrane stress. In (20), $\delta$ is a small perturbation parameter that transforms the perfect bifurcation equation into a perturbed bifurcation one. If one simplifies the wrinkling strain (9) as $\gamma^{\mathrm{wr}}(\mathrm{w})=\mathrm{Q}^{2} \mathrm{w}^{2} \mathrm{e}_{\mathrm{X}} \otimes \mathrm{e}_{\mathrm{X}}$ and if one combines (18) and (20), one can drop the deflection and deduce a nonlinear relation between membrane strain $\boldsymbol{\varepsilon}(\mathbf{u})$ and membrane stress $\mathbf{N}$ :

$$
\boldsymbol{\varepsilon}(u)+\frac{Q^{2} \delta^{2}}{\left(N_{X} Q^{2}+D\right)^{2}} \mathbf{e}_{X} \otimes \mathbf{e}_{X}=\left(\mathbf{L}^{m}\right)^{-1}: \mathbf{N} .
$$

\section{Some analytical and numerical solutions}

In this Part, we present few solutions of the system (17)(18)(19). Probably, many exact or approximated solutions of this new system can be found. In this paper, we limit ourselves, first to an analytical solution of the linearised system in order to establish the multiple scale character of membrane wrinkling, second to some numerical solutions of the full nonlinear problem (17)(18)(19) to show that this macroscopic model is able to describe the evolution of wrinkles even with a coarse finite element mesh.

\subsection{An analytical solution for wrinkling initiation}

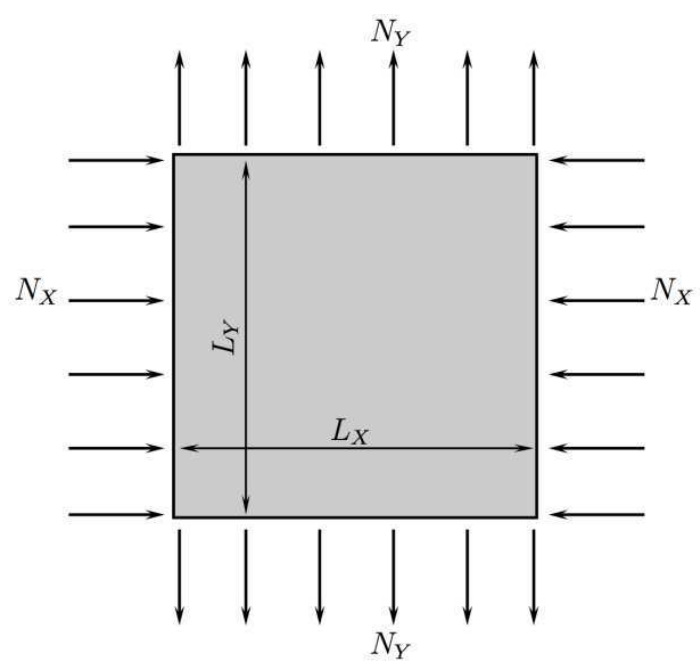

Figure 1: Rectangular membrane under biaxial load.

Let us consider the rectangular membrane $\left[0, L_{X}\right]_{X}\left[0, L_{Y}\right]$ pictured in Figure 1, which is submitted to a large uniform tensile stress $\mathrm{N}_{Y}=\mathrm{h} \sigma_{Y}>0$ and to a small uniform compressive stress loading $\mathrm{N}_{X}=\mathrm{h} \sigma_{X}<0$. The instability wavelength and the critical compressive stress will be deduced from the envelope equation (19). One seeks the value of the compressive stress at which wrinkling starts. The linearised version of the envelope equation (19) is rewritten as 
$-6 D Q^{2} \frac{\partial^{2} w}{\partial X^{2}}-\left(2 D Q^{2}+h \sigma_{Y}\right) \frac{\partial^{2} w}{\partial Y^{2}}+D Q^{4} w=h \mid \sigma_{X}\left(Q^{2} w-\frac{\partial^{2} w}{\partial Y^{2}}\right)$.

If the plate is clamped, it is known $[19,20]$ that the envelope $w$ vanishes on the boundary, what leads to a linear mode in the form:

$$
w(X, Y)=\sin \left(\pi X / L_{X}\right) \sin \left(\pi X / L_{Y}\right) .
$$

This leads to a classical relation between compressive stress and wavenumber.

$$
\left|\sigma_{X}\right|(Q)=\frac{6 D Q^{2} \frac{\pi^{2}}{L_{X}^{2}}+\left(2 D Q^{2}+h \sigma_{Y}\right) \frac{\pi^{2}}{L_{Y}^{2}}+D Q^{4}}{Q^{2}+\frac{\pi^{2}}{L_{X}^{2}}} .
$$

Hence our approach is able to define the stability wavenumber by minimizing the stress as a function of $Q$. For simplicity, we take into account the orders of magnitude $1<<Q L_{X}$, $2 D Q^{2} / h \approx\left|\sigma_{X}\right| \ll<\sigma_{Y}$ to simplify (22) in the following manner:

$$
\left|\sigma_{X}\right|(Q)=\frac{\sigma_{Y}}{Q^{2}} \frac{\pi^{2}}{L_{Y}^{2}}+\frac{D}{h} Q^{2} .
$$

The minimum of the latter yields values of the wavenumber and of the critical compressive stress that are consistent with the results of the literature [21][22]:

$$
\begin{aligned}
& Q^{w r}=\sqrt[4]{12 \pi^{2}\left(1-v^{2}\right)} \frac{1}{\sqrt{h L_{Y}}} \sqrt[4]{\frac{\sigma_{Y}}{E}} \cong 3.2 \frac{1}{\sqrt{h L_{Y}}} \sqrt[4]{\frac{\sigma_{Y}}{E}} \\
& l^{w r}=\frac{2 \pi}{Q^{w r} \cong 1.95 \sqrt{h L_{Y}}} \sqrt[4]{\frac{E}{\sigma_{Y}}} \\
& \left|\sigma_{X}^{w r}\right|=\frac{\pi}{\sqrt{3\left(1-v^{2}\right)}} \sqrt{E \sigma_{Y}} \frac{h}{L_{Y}}
\end{aligned}
$$

This simple calculation brings out the multiple scale character of the wrinkling phenomenon: indeed, the wrinkling threshold depends on the wavelength that is a microscopic quantity, but this wavelength depends of the width of the plate that is a macroscopic length. Thus a full wrinkling analysis has to associate micro and macro scales.

\subsection{A numerical post-bifurcation analysis for wrinkling}

The partial differential system (17) (18) (19) has been discretised by standard finite elements. The pure membrane approach (17)(21) has also been discretised in order to evaluate the importance of the spatial derivatives of the envelope. Eight nodes quadrangles Q8 have been chosen. The details of the procedure will be presented elsewhere. 
A thin rectangular membrane under uniaxial load (see Fig. 2) is studied, as in [23,24]. The side lengths $L_{x}$ and $L_{Y}$ are respectively $1400 \mathrm{~mm}$ and $200 \mathrm{~mm}$, and the thickness $h$ is $0.05 \mathrm{~mm}$. The long sides are stress-free. Along the short sides, a uniform tensile stress is applied in the axial direction and the displacements in the X-direction are locked.

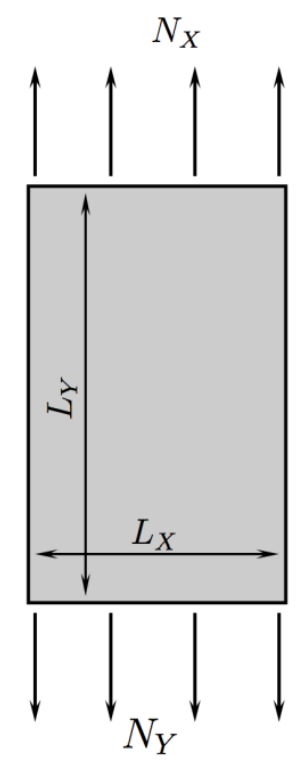

Figure 2. A rectangular membrane under uniaxial load.

Full nonlinear analyses of this problem have been done, first by a Q8 discretisation of the new model (17)(18)(19), second by a Q8 discretisation of the non-linear pure membrane model (17)(21), last by quadratic shell elements S825 of the Abaqus code that will be considered as the reference. The nonlinear problems associated with the first two models have been solved by the Asymptotic Numerical Method [25]. In Figure 3, one sees that the post-bifurcation patterns obtained by the new reduced model (17)(18)(19) are quite similar as those provided by the full shell model. This establishes the relevance of this new reduced model to represent the wrinkling modes in a case with a non uniform pre-buckling stress field.

In Figure 4, we have plotted the maximal deflection as a function of the applied tensile load for these three models. One sees that the new reduced model (17)(18)(19) gives about the same bifurcation point as the reference model as well as the post-bifurcation response. As expected, the number of unknowns is much smaller with the envelope models that do not describe explicitly the full details of the wrinkles. On the contrary, the pure membrane model underestimates the wrinkling threshold and overestimates the wrinkling amplitude. This is consistent with the analytical study of $\$ 3.1$ that has pointed out that the bifurcation load depends strongly on a macroscopic length and this influence of the macroscopic structure cannot be accounted if one neglects the spatial derivatives of the envelope $w(X, Y)$. 


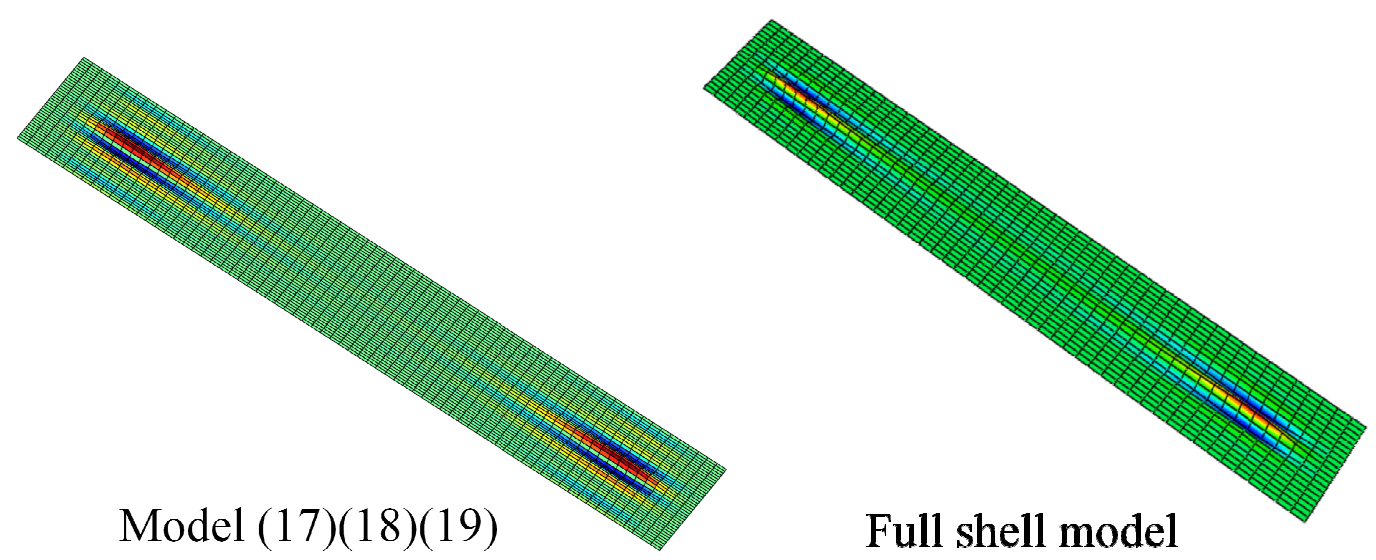

Figure 3. Post-bifurcation patterns obtained with the envelope model and a full shell model.

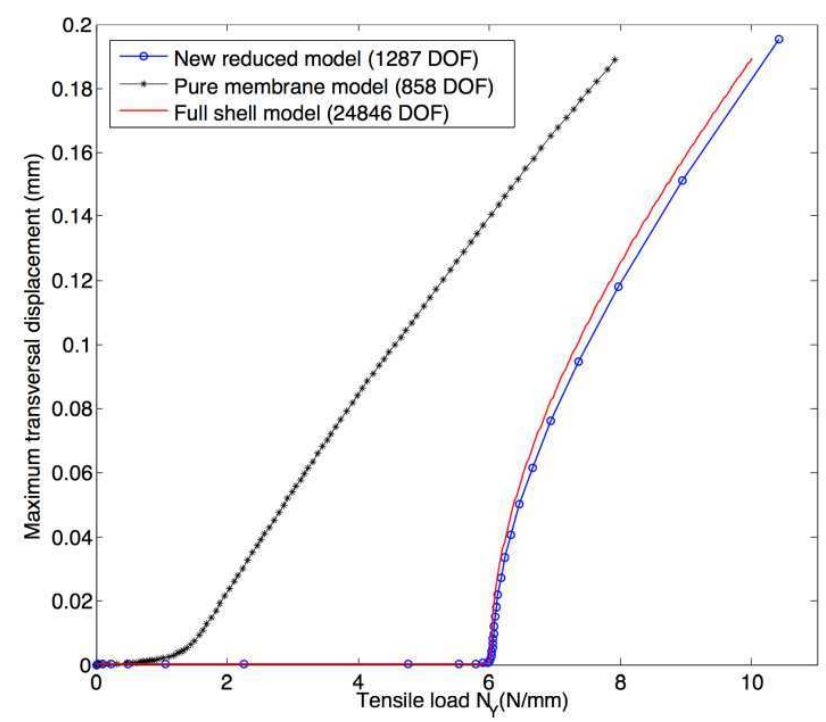

Figure 4. Bifurcation curves for the tensile problem of Figure 3, with three different models.

\section{Last comments}

A new wrinkling model has been presented in this paper. The approach is analog to bifurcation analyses for instability patterns via Landau-Ginzburg theory. The final model (17) (18) (19) is simple: the first equations (17) (18) are membrane equations including an additional wrinkling membrane strain and they are coupled with a sort of LandauGinzburg envelope equation (19). For this first paper about this new macroscopic approach of membrane wrinkling, two solutions have been presented. First an analytic solution of a clamped rectangular membrane illustrates that membrane wrinkling is a multi-scale problem that requires consistent multi-scale approaches. Second a numerical solution in a case with a non uniform pre-buckling stress has shown the relevance of the new reduced model and the necessity of accounting some spatial derivatives of the envelope. Likely this approach can have alternative applications, for instance for thin films on a compliant substrate [26] or for flatness defects induced by rolling process [27], but this could require some modifications to account for variable wrinkle orientations. 


\section{Acknowledgement}

The two last authors gratefully acknowledge the financial support of the National Research Fund Luxembourg (WRINKLE, Grant No. FNR/784868).

\section{References}

[1] Y.W. Wong, S. Pellegrino, Wrinkled membranes-Part1: experiments, J. Mech. Mater. Struct. 1 (2006) 3-25.

[2] C.G. Wang, X.W. Du, H.F. Tan, X.D. He, A new computational method for wrinkling analysis of gossamer space structures, Int. J. Solids Struct. 46 (2009) 1516-1526.

[3] Y. Lecieux, R. Bouzidi, Experimentation analysis on membrane wrinkling under biaxial load-Comparison with bifurcation analysis, Int. J. Solids Struct. 47 (2010) 2459-2475.

[4] Y. Lecieux, R. Bouzidi, Numerical wrinkling prediction of thin hyperelastic structures by direct energy minimization, Adv Eng Softw 50 (2012) 57-68.

[5] B. Tabarrok, Z. Qin, Nonlinear analysis of tension structures, Comput. Struct. 45 (1992) 973-984.

[6] J. Rodriguez, G. Rio, J.M. Cadou, J. Troufflard, Numerical study of dynamic relaxation with kinetic damping applied to inflatable fabric structures with extensions for 3D solid element and non-linear behavior, Thin Wall Struct. 49 (2011) 1468-1474.

[7] D.G. Roddeman, C.W.J. Oomens, J.D. Janssen, J. Drukker, The wrinkling of thin membranes: Part 1-theory, J. Appl. Mech. 54 (1987) 884-887.

[8] K. Lu, M. Accorsi, J. Leonard, Finite element analysis of membrane wrinkling, Int. J. Numer. Meth. Eng. 50 (2001) 1017-1038.

[9] H. Schoop, L. Taenzer, J. Hornig, Wrinkling of nonlinear membranes, Comput. Mech. 29 (2002) 68-74.

[10] Y. Miyazaki, Wrinkle/slack model and finite element dynamics of membrane, Int. J. Numer. Meth. Eng. 66 (2006) 1179-1209.

[11] T. Akita, T. Nakashino, M.C. Natori, K. C. Park, A simple computer implementation of membrane wrinkle behaviour via a projection technique, Int. J. Numer Meth. Eng. 71 (2007) 1231-1259.

[12] B. Banerjee, A. Shaw, D. Roy, The theory of Cosserat points applied to the analyses of wrinkled and slack membranes, Comput. Mech. 43 (2009) 415-429.

[13] N.A. Pimprikar, B. Banerjee, D. Roy, R.M. Vasu, S.R. Reid, New computational approaches for wrinkled and slack membranes, Int. J. Solids Struct. 47 (2010) 2476-2486.

[14] J.E. Wesfreid, S. Zaleski, (Editors), Cellular Structures in instabilities, Lecture Notes in Physics (210), Springer-Verlag, Heidelberg, 1984.

[15] R. Hoyle, Pattern formation, an introduction to methods, Cambrige University Press, 2006.

[16] N. Damil, M. Potier-Ferry, A generalized continuum approach to describe instability pattern formation by a multiple scale analysis, C. R. Mecanique 334 (2006) 674-678.

[17] N. Damil, M. Potier-Ferry, Influence of local wrinkling on membrane behaviour: a new approach by the technique of slowly variable Fourier coefficients, J. Mech. Phys. Solids 58 (2010) 1139-1153.

[18] H. Hu, N. Damil, M. Potier-Ferry, A bridging technique to analyze the influence of boundary conditions on instability patterns, J. Comput. Phys. 230 (2011) 3753-3764.

[19] K. Mhada, B. Braikat, H. Hu, N. Damil, M. Potier-Ferry, About macroscopic models of instability pattern formation, Int. J. Solids Struct. 49 (2012) 2978-2989. 
[20] Y. Liu, K. Yu, H. Hu, S. Belouettar, M. Potier-Ferry, A Fourier-related double scale analysis on instability phenomena of sandwich beams, Int. J. Solids Struct. 49 (2012) 3077-3088.

[21] E. Cerda, L. Mahadevan, Geometry and Physics of Wrinkling, Phys. Rev. Lett. 90 (2003) 074302-1-4.

[22] E. Cerda, K. Ravi-Chandar, L. Mahadevan, Wrinkling of an elastic sheet under tension, Nature 419 (2000) 579-580.

[23] N. Friedl, F.G. Rammerstorfer, F.D. Fisher, Buckling of stretched strips, Comput. Struct. 78 (2000) 185-190.

[24] N. Jacques, M. Potier-Ferry, On mode localisation in tensile plate buckling, C.R. Mecanique 333 (2005) 804-809.

[25] B. Cochelin, N. Damil, M. Potier-Ferry, Asymptotic-numerical methods and Padé approximants for nonlinear elastic structures, Int. J. Numer. Methods Eng. 37 (1994) 1187-1213.

[26] B. Audoly, A. Boudaoud, Buckling of a stiff film bound to a compliant substrate-Part II: A global scenario for the formation of herringbone pattern, J. Mech. Phys. Solids 56 (2008) 2422-2443.

[27] S. Abdelkhalek, P. Montmitonnet, M. Potier-Ferry, H. Zahrouni, N. Legrand, P. Buessler, Strip flatness modelling including buckling phenomena during thin strip cold rolling, Ironmak. Steelmak. 37 (2010) 290-297. 